\title{
REMARKS ON NON-LINEAR SCHRÖDINGER EQUATION WITH MAGNETIC FIELDS
}

\author{
LAURENT MICHEL
}

\begin{abstract}
We study the non-linear Schödinger equation with time depending magnetic field without smallness assumption at infinity. We obtain some results on the Cauchy problem, WKB asymptotics and instability.
\end{abstract}

\section{INTRODUCTION}

We consider the non-linear Schrödinger equation with magnetic field on $\mathbb{R}^{n}$

$$
i \partial_{t} u=\mathrm{H}_{\mathrm{A}(\mathrm{t})} u-b^{\gamma} f(x, u)
$$

with initial condition

$$
u_{\mid t=t_{0}}=\varphi
$$

Here

$$
\mathrm{H}_{\mathrm{A}(\mathrm{t})}=\sum_{j=1}^{n}\left(i \partial_{x_{j}}-b A_{j}(t, x)\right)^{2}, t \in \mathbb{R}, \quad x \in \mathbb{R}^{n}
$$

is the time-depending Schrödinger operator associated to the magnetic potential $\left.A(t, x)=\left(A_{1}(t, x), \ldots, A_{n}(t, x)\right), b \in\right] 0,+\infty[$ is a parameter quantizing the strength of the magnetic field and $\gamma \geq 0$. We sometimes omit the space dependence and write $A(t)$ instead of $A(t, x)$. The aim of this note is to show that recent improvement in the analysis of non-linear Schrödinger equations can be adapted to the case with magnetic field. As an important preliminary, we study the local Cauchy problem for (1.1) in energetic space. Let us begin with the general framework of our study.

We suppose that the magnetic potential is a smooth function $A \in C^{\infty}\left(\mathbb{R}_{t} \times\right.$ $\mathbb{R}_{x}^{n}, \mathbb{R}^{n}$ ) and that it satisfies the following assumption.

$$
\begin{aligned}
& \text { Assumption 1. (1) } \forall \alpha \in \mathbb{N}^{n} \sup _{(t, x) \in \mathbb{R} \times \mathbb{R}^{n}}\left|\partial_{x}^{\alpha} \partial_{t} A\right| \leq C_{\alpha} . \\
& \text { (2) } \forall|\alpha| \geq 1, \sup _{(t, x) \in \mathbb{R} \times \mathbb{R}^{n}}\left|\partial_{x}^{\alpha} A\right| \leq C_{\alpha} . \\
& \text { (3) } \exists \epsilon>0, \forall|\alpha| \geq 1, \sup _{(t, x) \in \mathbb{R} \times \mathbb{R}^{n}}\left|\partial_{x}^{\alpha} B\right| \leq C_{\alpha}\langle x\rangle^{-1-\epsilon}
\end{aligned}
$$

where $B(t, x)$ is the matrix defined by $B_{j k}=\partial_{x_{j}} A_{k}-\partial_{x_{k}} A_{j}$.

Remark that compactly supported perturbations of linear (with respet to $x$ ) magnetic potentials satisfy the above hypothesis.

Under Assumption 10 the domain $D\left(\mathrm{H}_{\mathrm{A}(\mathrm{t})}\right)=\left\{u \in L^{2}\left(\mathbb{R}_{x}^{n}\right), \mathrm{H}_{\mathrm{A}(\mathrm{t})} u \in L^{2}\left(\mathbb{R}_{x}^{n}\right)\right\}$ does not depend on $t$. Indeed, for $t, t^{\prime} \in \mathbb{R}$ one has

$$
\mathrm{H}_{\mathrm{A}\left(\mathrm{t}^{\prime}\right)}=\mathrm{H}_{\mathrm{A}(\mathrm{t})}+b W\left(t, t^{\prime}\right)\left(i \nabla_{x}-b A(t)\right)+b\left(i \nabla_{x}-b A(t)\right) W\left(t, t^{\prime}\right)+b^{2} W\left(t, t^{\prime}\right)^{2}
$$

Key words and phrases. Schrödinger equation, Magnetic fields, Strichartz estimate. 
with $x \mapsto W\left(t, t^{\prime}, x\right)=\int_{t}^{t^{\prime}} \partial_{s} A(s, x) d s$ bounded as well as its $x$-derivatives uniformly with respect to $t, t^{\prime}$ in any compact set. In fact, the above identity shows that the space

$$
H_{m g}^{\beta}\left(\mathbb{R}^{n}\right)=\left\{u \in L^{2}\left(\mathbb{R}^{n}\right),\left(1+\mathrm{H}_{\mathrm{A}(\mathrm{t})}\right)^{\beta / 2} u \in L^{2}\left(\mathbb{R}^{n}\right)\right\}
$$

does not depend on $t \in \mathbb{R}$. As $D\left(\mathrm{H}_{\mathrm{A}(\mathrm{t})}\right)=H_{m g}^{2}\left(\mathbb{R}^{n}\right)$, the above statement is straightforward. Moreover, the natural norms on this space are equivalent and this equivalence is uniform with respect to the parameter $b$ for close times. More precisely, denoting $m_{A}=\sup _{(t, x) \in \mathbb{R} \times \mathbb{R}^{n}}\left|\partial_{t} A(t, x)\right|$, we have the following

Proposition 1.1. Suppose that Assumption 1 is satisfied and let $\beta>0$ and $T>0$. Then, for all $t, t^{\prime} \in \mathbb{R}$ such that $\left|t-t^{\prime}\right| \leq b^{-1} T$ and all $u \in H_{m g}^{\beta}$ we have

$$
\left\|\left(\mathrm{H}_{\mathrm{A}\left(\mathrm{t}^{\prime}\right)}+1\right)^{\beta} u\right\|_{L^{2}} \leq\left(1+2 m_{A} T+m_{A}^{2} T^{2}\right)^{\beta}\left\|\left(\mathrm{H}_{\mathrm{A}(\mathrm{t})}+1\right)^{\beta} u\right\|_{L^{2}} .
$$

Proof. It is a straightforward consequence of equation (1.3), Assumption 1 and the fact that $\left(i \nabla_{x}-b A(t)\right)\left(\mathrm{H}_{\mathrm{A}(\mathrm{t})}+1\right)^{-1}$ is bounded by 1 in $L^{2}$.

For $\beta \in \mathbb{N}$ we set

$$
\|u\|_{H_{A(t)}^{\beta}}=\left\|\left(i \nabla_{x}-b A(t)\right)^{\beta} u\right\|_{L^{2}}+\|u\|_{L^{2}}
$$

This norm is clearly equivalent (uniformly with respect to $b$ ) to $\left\|\left(1+\mathrm{H}_{\mathrm{A}(\mathrm{t})}\right)^{\beta / 2} u\right\|_{L^{2}}$. In regard of Proposition 1.1 we define the magnetic Sobolev norm by

$$
\|u\|_{H_{m g}^{\beta}}=\|u\|_{H_{A\left(t_{0}\right)}^{\beta}} .
$$

Under Assumption 1 it is well-known (see [15], Th 4.6, p143 or [18]) that for $\varphi \in H_{m g}^{1}$, the linear Schrödinger equation

$$
i \partial_{t} u=\mathrm{H}_{\mathrm{A}(\mathrm{t})} u, \quad u_{\mid t=s}=\varphi
$$

has a solution $U_{0}(t, s) \varphi$. The operator $U_{0}(t, s)$ maps $H_{m g}^{1}$ into itself, is continuous from $L^{2}$ into $L^{2}$ and from $H_{m g}^{1}$ into $H_{m g}^{1}$. Moreover, $U_{0}(t, s) \varphi$ is the unique $H_{m g}^{1}$ valued solution of (1.5) and $U_{0}(t, s)$ is unitary.

The first aim of this paper is to solve the Cauchy problem for the non-linear equation in the most appropriate space. We state the assumptions on the nonlinearity $f$. We suppose that $f: \mathbb{R}^{n} \times \mathbb{C} \rightarrow \mathbb{C}$ is a measurable function such that

Assumption 2. (1) $f(x, 0)=0$ almost every where.

(2) $\exists M \geq 0, \alpha \in\left[0, \frac{4}{n-2}[(\alpha \in[0, \infty[\right.$ if $n=1,2)$ such that

$$
\mid f\left(x, z_{1}\right)-f\left(x, z_{2}\right)\left[\leq M\left(1+\left|z_{1}\right|^{\alpha}+\left|z_{2}\right|^{\alpha}\right)\left|z_{1}-z_{2}\right|\right.
$$

for almost all $x \in \mathbb{R}^{n}$ and all $z_{1}, z_{2} \in \mathbb{C}$.

(3) $\forall z \in \mathbb{C}, f(x, z)=(z /|z|) f(x,|z|)$

Remark that these assumptions are often used in the case $A=0$. More precisely, in the case $A=0$, the second property of the above assumption corresponds to a subcritical non-linearity with respect to $H^{1}$.

Let us introduce some energy functional associated to these non-linerarities. We define

$$
F(x, z)=\int_{0}^{|z|} f(x, s) d s, G(u)=\int_{\mathbb{R}^{n}} F(x, u(x)) d x
$$


and for $t \in \mathbb{R}$ and $u \in H_{m g}^{1}$ we define the energy

$$
E(b, t, u)=\int_{\mathbb{R}^{n}} \frac{1}{2}\left|\left(i \nabla_{x}-b A(t, x)\right) u(x)\right|^{2} d x-b^{\gamma} G(u) .
$$

Formally, it is not hard to see that any sufficiently regular solution of (1.1), (1.2), enjoys the following energy evolution law:

$$
E(b, t, u)=E(b, 0, \varphi)-\operatorname{Re} \int_{0}^{t}\left\langle\partial_{s} A(s) u(x),(i \nabla-A(s)) u(s)\right\rangle_{L^{2}} d s .
$$

Therefore, the natural space to solve (1.1), (1.2) seems to be $H_{m g}^{1}$.

Now we are in position to state our first result.

Theorem 1. Suppose that Assumptions 1 and 2 are satisfied and let $\varphi \in H_{m g}^{1}$. Then, there exists $T_{b}, T^{b}>0$ and a unique $u \in C(]-T_{b}, T^{b}\left[, H_{m g}^{1}\right) \cap C^{1}(]-$ $T_{b}, T^{b}\left[, H_{m g}^{-1}\right)$ solution of (1.1). Moreover, either $T_{b}=\infty$ (resp. $\left.T^{b}=\infty\right)$, or $\lim _{t \rightarrow-T_{b}}\|u(t)\|_{H_{m g}^{1}}=\infty\left(\right.$ resp. $\left.\lim _{t \rightarrow T^{b}}\|u(t)\|_{H_{m g}^{1}}=\infty\right)$ and

$$
E(b, t, u)=E(b, 0, \varphi)-\operatorname{Re} \int_{0}^{t}\left\langle\partial_{s} A(s) u(x),(i \nabla-A(s)) u(s)\right\rangle_{L^{2}} d s,
$$

for all $t \in]-T_{b}, T^{b}$. Additionally, there exists $\epsilon>0$ such that, for all $b>0$ and $\varphi \in H_{m g}^{1}$ such that $\|\varphi\|_{H_{m g}^{1}} \leq C b$, we have $T_{b}, T^{b} \geq \epsilon b^{-\delta}$ with $\delta=\max \left(1,2 \gamma, \frac{2 \gamma}{\alpha}\right)$.

Let us make a few remarks on this result. The Cauchy problem for non-linear Schrödinger equation has a long story. In absence of magnetic field there are numerous results; see for instance [9, 10, 5].

In presence of magnetic field, the behavior of $A$ when $|x|$ becomes large plays an important role. In the case where the magnetic potential $A$ is bounded, the spaces $H_{m g}^{1}$ and $H^{1}$ coincide and the Cauchy problem can be solved in $H^{1}$ using usual techniques. If the magnetic field is unbounded, it is not possible to solve the Cauchy problem in $H^{1}$ as multiplication by $A$ is not bounded on $L^{2}$.

To avoid this difficulty some authors work in the weighted Sobolev space $\Sigma=$ $\left\{u \in H^{1}\left(\mathbb{R}^{n}\right),(1+|x|) u \in L^{2},\right\}$ (see for instance [7, [14). In particular, they require some decay of the initial data at infinity.

In the case of [7, this decay is required because the author use dispersive properties for the Laplacian instead of $\mathrm{H}_{\mathrm{A}(\mathrm{t})}$. In 14 the author use magnetic Strichartz estimates but their method based on fixed-point theorem is not adapted to the magnetic context and requires decay of the solution at infinity.

On the other hand, there exists also of a result of Cazenave and Esteban [4] dealing with the special case where the magnetic field $B$ is constant (and hence, $A$ is linear with respect to $x$ ). In a way, this paper is more satisfactory as they need only $u_{0}$ to belong to the energy space. Nevertheless, their result applies only to constant magnetic field.

Our theorem is, then a generalization of the above results. Before going further, let us remark that for unbounded $A$, the spaces $H^{1}, H_{m g}^{1}$ and $\Sigma$ are different. First, it is evident that $\Sigma$ is contained in $H^{1} \cap H_{m g}^{1}$. Let us give an example where $\Sigma$ is strictly contained in $H_{m g}^{1}$. For this purpose, we restrict ourseleves to the case where the dimension $n=2$ and consider the magnetic potential $A(x, y)=(y, x)$. 
Let $g \in H^{1}\left(\mathbb{R}^{2}\right)$ be such that $|x| g \notin L^{2}$, then a simple calculus shows that $f(x, y)=$ $g(x, y) e^{-i x y}$ belongs to $H_{m g}^{1} \backslash \Sigma$.

In the case of defocusing non-linearities the energy law implies the following result.

Corollary 1.2. Suppose that $F(x, z) \leq 0$ for all $x, z$, then $T_{b}, T^{b}=+\infty$.

Proof. For $F \leq 0$, we deduce from (1.7) and Cauchy-Schwarz inequality, that

$$
\|(i \nabla-A(t)) u(t)\|_{L^{2}} \leq C_{1}+C_{2} \int_{0}^{t}\|(i \nabla-A(s)) u(s)\|_{L^{2}} d s
$$

for some fixed constant $C_{1}, C_{2}>0$. Hence, Gronwall Lemma shows that $\|(i \nabla-$ $A(t)) u(t) \|_{L^{2}}$ remains bounded on any bounded time-interval. Using (1.6) and the characterization of $T_{b}$, we obtain the result.

The next section contains the proof of Theorem 1. In section 3 we give some qualitative results on the solution of (1.1) in the limit $b \rightarrow \infty$. More precisely, we can construct WKB solutions and prove instability results with respect to initial data and parameter $b$.

\section{Cauchy problem in the energy space}

The proof of theorem 11 relies on the Strichartz estimates proved in [18] for the problem

$$
i \partial_{t} u=\mathrm{H}_{\mathrm{A}(\mathrm{t})} u+g(t), \quad u_{\mid t=s}=\varphi
$$

Theorem 2. (Yajima) Let $I$ be a finite real interval, $(q, r)$ and $\left(\gamma_{j}, \rho_{j}\right), j=1,2$ be such that $r, \rho_{j} \in\left[2, \frac{2 n}{n-2}\left[, \frac{2}{q}=n\left(\frac{1}{2}-\frac{1}{r}\right)\right.\right.$ and $\frac{2}{\gamma_{j}}=n\left(\frac{1}{2}-\frac{1}{\rho_{j}}\right)$. Let $g_{j} \in$ $L^{\gamma_{j}^{\prime}}\left(I, L^{\rho_{j}^{\prime}}\left(\mathbb{R}_{x}^{n}\right)\right), j=1,2$, where $\gamma_{j}^{\prime}, \rho_{j}^{\prime}$ are the conjugate exposant of $\gamma_{j}, \rho_{j}$. Then the solution $u$ to (2.1) with $g=g_{1}+g_{2}$ satisfies

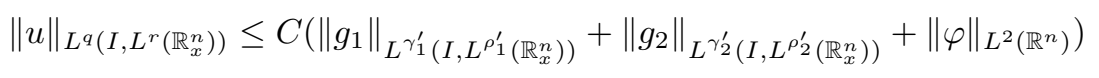

where the constant $C$ depends only on the length of $I$ and the constant $C_{\alpha}$ of Assumption 1 .

Proof. In the case $g=0$ it is exactly Theorem 1 of [18. In the general case it suffices to work as in the proof of Proposition 2.15 of [2] using a celebrated result of Christ and Kiselev [6. The fact that the constant $C$ depends only on the $C_{\alpha}$ is a direct consequence of the construction of Yajima 18 .

Remark 2.1. In the case where the magnetic potential is not regular, there are some recent results of A. Stefanov [16] and Georgiev-Tarulli [8] which provide Strichartz estimates under smallness assumption on the magnetic fields. This should lead to the corresponding existence and uniqueness result for $N L S$ in the case of small magnetic field. This could also have consequences on the well-posedness of the Schrödinger-Maxwell system (see [12], 13], 17] for results on this topics).

It is important to notice that Theorem 1 is not a straightforward consequence of the above Strichartz estimate. Indeed, if we try to apply a fixed point method to equation (1.1), a problem occurs when we try to control the norm of the nonlinearity in the $H_{m g}^{1}$ norm. Consider for instance the case $f(u)=|u|^{2} u$, then

$$
\left(i \nabla_{x}-b A(t)\right)\left(|u|^{2} u\right)=|u|^{2}\left(i \nabla_{x}-b A(t)\right)(u)+u i \nabla_{x}\left(\left|u^{2}\right|\right) .
$$


The first therm of the right hand side of this equality will be controlled by $\|u\|_{H_{m q}^{1}}$, whereas in the second term, as $A(t, x)$ is not bounded with respect to $x$, there is no chance to control $i \nabla_{x}\left(\left|u^{2}\right|\right)$ by $\left(i \nabla_{x}-b A(t)\right)\left(\left|u^{2}\right|\right)$. For the same reason it does not seem easy to solve the Cauchy problem in magnetic Sobolev spaces of high degree.

To overcome this difficulty, we work as in [5], 4] and approximate the solution of (1.1) by solution of a non-linear Schrödinger equation with non-linearity linearized at infinity. In the work of Cazenave and Weissler, the main tool to justify the approximation is an energy conservation. In our case, the Hamiltonian depends on time, so that the energy is not conserved. Nevertheless, the error term is controled by the $H_{m g}^{1}$-norm so that it is possible to implement the same strategy. Another difference involved by the dependance with respect to time of the Hamiltonian is that usual techniques to solve the Cauchy problem with regular initial data and nice non-linearities can not apply in our context. Therefore, additionnaly to the approximation of the non-linearity, we have to introduce an approximation of the magnetic field itself and justify the convergence to our initial problem.

Let us introduce the approximated nonlinearities used in the sequel. Following [5], we decompose $f=\tilde{f}_{1}+\tilde{f}_{2}$ with

$$
\tilde{f}_{1}(x, z)=1_{\{|z| \leq 1\}} f(x, z)+1_{\{|z| \geq 1\}} f(x, 1) z
$$

and

$$
\tilde{f}_{2}(x, z)=1_{\{|z| \geq 1\}}(f(x, z)-f(x, 1) z) .
$$

Next we define $f_{m}=\tilde{f}_{1}+\tilde{f}_{2, m}$ where

$$
\tilde{f}_{2, m}(x, z)=1_{\{|z| \leq m\}} \tilde{f}_{2}(x, z)+1_{\{|z| \geq m\}} \tilde{f}_{2}(x, m) \frac{z}{m}
$$

Remark that these functions satisfy Assumption 2, We consider also the energy functional associated to these approximated non-linearities. We define

$$
F_{m}(x, z)=\int_{0}^{|z|} f_{m}(x, s) d s, G_{m}(u) \int_{\mathbb{R}^{n}} F_{m}(x, u(x)) d x
$$

and for $t \in \mathbb{R}$ and $u \in H_{m g}^{1}$ we set

$$
E_{m}(b, t, u)=\int_{\mathbb{R}^{n}} \frac{1}{2}\left|\left(i \nabla_{x}-b A(t, x)\right) u(x)\right|^{2} d x-G_{m}(u) .
$$

Finally, we remark that replacing the magnetic potential $A(t, x)$ by $A\left(t+t_{0}, x\right)$ it suffices to prove Theorem 1 for $t_{0}=0$.

On the other hand, to enlight the notations we prove the theorem in the particular case $b=1$. To get the general case it suffices to keep track of $b$ along the proof. We will also restrict our study to $t \geq 0$, the other case being treated by reversing time in the equation.

2.1. Preliminary results. In the sequel, we will need Sobolev embeddings in the magnetic context. In this subsection, $A$ is a magnetic potential satisfying Assumption 1

Lemma 2.2. Let $0<s<\frac{n}{2}$ and $p_{s}=\frac{2 n}{n-2 s}$, then $H_{A}^{s}$ is continuously embedded in $L^{p}\left(\mathbb{R}^{n}\right)$ for all $p \in\left[2, p_{s}\right]$ and there exists $C>0$ independent of $A$ such that

$$
\|u\|_{L^{p}} \leq C\|u\|_{H_{A}^{s}}
$$


Proof. From the diamagnetic inequality (see [1]), we know that almost everywhere we have

$$
|u|=\left|\left(\mathrm{H}_{\mathrm{A}}+1\right)^{-\frac{s}{2}}\left(\mathrm{H}_{\mathrm{A}}+1\right)^{\frac{s}{2}} u\right| \leq(-\Delta+1)^{-\frac{s}{2}}\left|\left(\mathrm{H}_{\mathrm{A}}+1\right)^{\frac{s}{2}} u\right| .
$$

Taking the $L^{p}$ norm, the result follows from standard Sobolev inequalities.

Next we prove a technical result on the non-linearity.

Proposition 2.3. Let $M>0, r_{1}=\rho_{1}=2$ and $r_{2}=\rho_{2}=\alpha+2$ then

(1) the sequence $\left(\tilde{f}_{2, m}(., u)\right)_{m \in \mathbb{N}^{*}}$ converges to $\tilde{f}_{2}(., u)$ in $L^{\rho_{2}^{\prime}}\left(\mathbb{R}^{n}\right)$ uniformly with respect to $u \in H_{A}^{1}$ such that $\|u\|_{H_{A}^{1}} \leq M$.

(2) there exists $C(M)>0$ independent of $A$ such that for all $m \in \mathbb{N}^{*}$ and for all $u, v \in H_{A}^{1}$ with $\max \left(\|u\|_{H_{A}^{1}},\|v\|_{H_{A}^{1}}\right) \leq M$ we have

$$
\begin{gathered}
\left\|\tilde{f}_{1}(., u)-\tilde{f}_{1}(., v)\right\|_{L^{\rho_{1}^{\prime}\left(\mathbb{R}^{n}\right)}} \leq C(M)\|u-v\|_{L^{r_{1}}} \\
\left\|\tilde{f}_{2, m}(., u)-\tilde{f}_{2, m}(., v)\right\|_{L^{\rho_{2}^{\prime}\left(\mathbb{R}^{n}\right)}}+\left\|\tilde{f}_{2}(., u)-\tilde{f}_{2}(., v)\right\|_{L^{\rho_{2}^{\prime}\left(\mathbb{R}^{n}\right)}} \leq C(M)\|u-v\|_{L^{r_{2}\left(\mathbb{R}^{n}\right)}}
\end{gathered}
$$

Proof. We follow the method of Example 3 in [5]. Taking $\chi$ the characteristic function of the set $\left\{x \in \mathbb{R}^{n}|| u(x) \mid>m\right\}$ and using Assumption 2, we have

$$
\left\|\tilde{f}_{2}(u)-\tilde{f}_{2, m}(u)\right\|_{L^{\rho_{2}^{\prime}\left(\mathbb{R}^{n}\right)}} \leq 2\left\|\chi|u|^{\alpha+1}\right\|_{L^{\rho_{2}^{\prime}}}=2\|\chi u\|_{L^{\alpha+2}}^{\alpha+1} .
$$

On the other hand, using Hölder inequality and Lemma 2.2 we get for $p=\frac{2 n}{n-2}$,

$$
\|u\|_{H_{A}^{1}} \geq C\|\chi u\|_{L^{p}} \geq C m^{1-\frac{\alpha+2}{p}}\|\chi u\|_{L^{\alpha+2}}^{\frac{\alpha+2}{p}} .
$$

As $\alpha<\frac{4}{n-2}$ then $1-\frac{\alpha}{p+2}>0$. Combining equations (2.9) and (2.9), we obtain the first point of the proposition.

The second assertion follows, as in example 3 in [5], from Hölder inequality, Assumption 22 and Lemma 2.2. The fact that the constant $C(M)$ is independent of the magnetic fields follows from the uniformity of the constant in Lemma 2.2

Lemma 2.4. Let $T>0$ and $\gamma_{k}, k=1,2$ be defined by $\frac{2}{\gamma_{k}}=n\left(\frac{1}{2}-\frac{1}{\rho_{k}}\right)$. For $M>0$ there exists a constant $C(M)$ independent of $A$, such that for all $u, v \in H_{A}^{1}$ with $\|u\|_{H_{A}^{1}} \leq M$ and $\|v\|_{H_{A}^{1}} \leq M$ we have

$$
|G(u)-G(v)|+\left|G_{m}(u)-G_{m}(v)\right| \leq C(M)\left(\|v-u\|_{L^{2}}+\|v-u\|_{L^{2}}^{\nu}\right),
$$

with $\frac{2}{\nu}=\frac{n}{2}-\frac{n}{\alpha+2}$ and for all $u, v \in L^{\infty}\left([0, T] H_{A}^{1}\right)$,

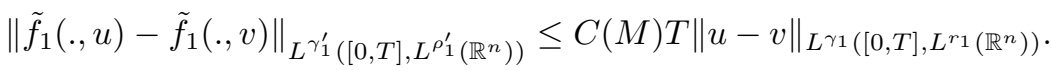

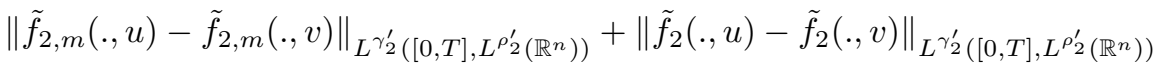

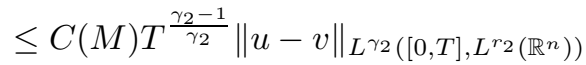

Moreover, $G_{m} \rightarrow G$ as $m \rightarrow \infty$ uniformly on bounded sets of $H_{A}^{1}$.

Proof. Remark that $G(u)=\int_{0}^{1}\langle f(x, s u), u\rangle_{L^{2}} d s$ and $G_{m}(u)=\int_{0}^{1}\left\langle f_{m}(x, s u), u\right\rangle_{L^{2}} d s$ and copy the proof of Lemma 3.3 in [5], replacing classical Sobolev inequalities by Lemma 2.2 and using Proposition 2.3.

We are now in position to prove the uniqueness part of Theorem 1

Proposition 2.5. Let $T>0$ and $u, v \in C\left(\left[0, T\left[, H_{m g}^{1}\right) \cap C^{1}\left(\left[0, T\left[, H_{m g}^{-1}\right)\right.\right.\right.\right.$ be solution of (1.1). Then $u=v$. 
Proof. Let $u, v \in C\left(\left[0, T\left[, H_{m g}^{1}\right) \cap C^{1}\left(\left[0, T\left[, H_{m g}^{-1}\right)\right.\right.\right.\right.$ be solution of (1.1), and set $w=v-u$. Then $w(0)=0$ and

$$
i \partial_{t} w-\mathrm{H}_{\mathrm{A}(\mathrm{t})} w=\widetilde{f}_{1}(u)-\widetilde{f}_{1}(v)+\widetilde{f}_{2}(u)-\widetilde{f}_{2}(v) .
$$

Let $r \in\left[2, \frac{2}{n-2}\right]$ and $q>2$ such that $\frac{2}{q}=n\left(\frac{1}{2}-\frac{1}{r}\right)$. Apply Theorem 2 together with Lemma 2.4, we get

$$
\|w\|_{L^{q}\left(\left[0, T\left[L^{r}\right)\right.\right.} \leq C\left(T+T^{\gamma_{2}}\right)\left(\|w\|_{L^{\infty}\left(\left[0, T\left[L^{2}\right)\right.\right.}+\|w\|_{L^{\gamma_{2}\left(\left[0, T\left[, L^{\rho_{2}}\right)\right.\right.}}\right)
$$

where $\gamma_{2}=\frac{\alpha+1}{\alpha+2}$ and $\frac{2}{\gamma_{2}}=n\left(\frac{1}{2}-\frac{1}{\rho_{2}}\right)$. As we can alternatively take $(q, r)$ to be equal to $(2, \infty)$ and $\left(\gamma_{2}, \rho_{2}\right)$, we get the announced result by summing the obtained inequalities and making $T>0$ small enough.

2.2. Autonomous case. In this section we explain briefly how to solve the Cauchy problem in $H_{m g}^{1}$ when the magnetic field $A(t, x)=A(x)$ is time independent. In this context, the functional $E$ does not depend on time and formally we have the following conservation of energy. Suppose that $u$ is solution of (1.1) then

$$
E(b, u(t))=E(b, \varphi), \forall t .
$$

More precisely, we prove the following

Proposition 2.6. Let $M>0$ and $C_{\alpha}, \alpha \in \mathbb{N}^{n}$ a family of finite positive numbers. There exists $T>0$ depending only on $M$ and the $C_{\alpha}$ such that for all $A$ satisfying $\partial_{t} A=0$ and Assumption 1 with $C_{\alpha}$ and for all $\varphi \in H_{A}^{1}$ such that $\|\varphi\|_{H_{A}^{1}} \leq M$, there exists a unique $u \in C^{0}\left(\left[0, T\left[, H_{A}^{1}\right) \cap C^{1}(] 0, T\left[, H_{A}^{-1}\right)\right.\right.$ maximal solution of

$$
i \partial_{t} u=\mathrm{H}_{\mathrm{A}} u+f(x, u)
$$

with initial condition $u_{\mid t=0}=\varphi$. Moreover, for all $t \in[0, T[$ we have

$$
E(b, u(t))=E(b, \varphi),
$$

and if $T<\infty$ then $\lim _{t \rightarrow T}\|u\|_{H_{A}^{1}}=\infty$.

The proof is slight adaption of [5], 4, to our context. We need also to investigate the dependence of the existence time with respect to the magnetic field. However, the scheme of proof is the same and consists to consider an approximate problem and justify convergence on fixed time intervals. Let us give the main steps of the proof.

Step 1. Let $f_{m}$ be defined by (2.3), (2.4), (2.5) and let $A$ be a magnetic field satisfying the above hypotheses. Consider the problem

$$
i \partial_{t} u=\mathrm{H}_{\mathrm{A}} u+f_{m}(x, u), \quad u_{t=0}=\varphi
$$

with $\varphi \in H_{A}^{1}$. We have the following

Lemma 2.7. Let $\varphi \in H_{A}^{1}$, then there exists $\tau_{m, A}>0$ such that there exists $u_{m} \in$ $C\left(\left[0, \tau_{m, A}\left[, H_{A}^{1}\right) \cap C^{1}\left(\left[0, \tau_{m, A}\left[, H_{A}^{-1}\right)\right.\right.\right.\right.$ solution of [2.11). Moreover we have for all $t \in\left[0, \tau_{m, A}[\right.$,

$$
E_{m}\left(u_{m}\right)=E_{m}(\varphi)
$$

and

$$
\left\|u_{m}(t)\right\|_{L^{2}}=\|\varphi\|_{L^{2}}
$$


Proof. The proof is the same as that of Lemma 3.5 of [5], replacing usual derivatives by magnetic derivatives.

Step 2. We show that the existence time $\tau_{m, A}$ can be bounded from below uniformly with respect to $m \in \mathbb{N}$ and $A$ satisfying Assumptions of the above proposition.

Lemma 2.8. Let $M>0$. There exists $T_{1}=T_{1}(M)>0$ such that for all $m \in \mathbb{N}$, all A satisfying Assumption 1 and all $\varphi \in H_{A}^{1}$ with $\|\varphi\|_{H_{A}^{1}} \leq M$ we have

$$
\left\|u_{m}\right\|_{L^{\infty}\left(\left[0, T_{1}\right], H_{A}^{1}\right)} \leq 2\|\varphi\|_{H_{A}^{1}} .
$$

Proof. The proof is exactly the same as in Lemma 3.6 of [5], making use of Lemma 2.7 (in particular, we use strongly the conservation of energy) and Proposition 2.3 to get uniformity with respect to $A$.

Step 3. The final step is to prove convergence of the $u_{m}$ to solution of the initial problem. First we prove convergence in $L^{2}$.

Lemma 2.9. Let $M>0$ and $C_{\alpha}, \alpha \in \mathbb{N}^{n}$ a family of finite positive numbers. There exists $T_{2}>0$ depending only on $M$ and the $C_{\alpha}$ such that for all $A$ satisfying Assumption 1 with $C_{\alpha}$ and for all $\varphi \in H_{A}^{1}$ such that $\|\varphi\|_{H_{A}^{1}} \leq M$, such that $\left(u_{m}\right)_{m \in \mathbb{N}}$ is a Cauchy sequence in $C\left(\left[0, T_{2}\right], L^{2}\right)$.

Proof. The proof is the same as in [5, making use of Theorem 2. Lemma 2.4] Proposition 2.3 and Lemma 2.7

Now, we can complete the proof of Theorem 1, We denote $u$ the limit of $u_{m}$ in $C\left(\left[0, T_{2}\right], L^{2}\right)$. From Lemma 2.8 , it follows that $u \in L^{\infty}\left(\left[0, T_{2}\right], H_{A}^{1}\right)$ and by Lemma 2.2, $u_{m}$ converges to $u$ in $C\left(\left[0, T_{2}\right], L^{r}\right)$ for all $r \geq 2 n /(n-2)$. Hence, it follows from Proposition 2.3 that $f_{m}\left(u_{m}\right)$ converges to $f(u)$ in $C\left(\left[0, T_{2}\right], H_{A}^{-1}\right)$ and $u$ solves 1.1 in $L^{\infty}\left(\left[0, T_{2}\right], H_{A}^{-1}\right)$. Moreover, combining Lemma 2.4 and 2.7 we prove that

$$
E(b, t, u)=E(b, 0, \varphi) .
$$

This shows that $u \in C\left(\left[0, T_{2}\right], H_{m g}^{1}\right)$ and hence $u \in C^{1}\left(\left[0, T_{2}\right], H_{A}^{-1}\right)$.

2.3. Cauchy problem in the time-depending case. We suppose now that $A(t, x)$ satisfies Assumption 1. The strategy of proof is the same as in autonomous case and we first consider the problem

$$
i \partial_{t} u=\mathrm{H}_{\mathrm{A}(\mathrm{t})} u+f_{m}(x, u), \quad u_{t=0}=\varphi
$$

At least formally, we can see that the energy of the solution of this equation satisfies the following rule

$$
E(t, u)=E(0, \varphi)-\operatorname{Re} \int_{0}^{t}\left\langle\partial_{s} A(s) u(s),\left(i \nabla_{x}-A(s)\right) u(s)\right\rangle d s .
$$

This will replace the energy conservation in our approach. On the other hand another problem occurs if we try to apply the proof of [5]. Indeed, the first step should be to obtain a generalization of Lemma 2.7 in the time depending framework. Following the proof of Lemma 3.5 in [5], we should regularize the initial data and solve the Cauchy problem in $H_{m g}^{2}$. The issue is that contrary to the autonomous case, the existence of smooth solution is not easy to prove. Indeed, the key point in the approach of $\left[5\right.$ is that for any $g \in C\left([0, T], H^{1}\right)$ Lipschitz continuous with respect to time, the function $v(t)=\int_{0}^{t} U_{0}(t, s) g(s) d s$ is also Lipschitz continuous with respect to time. Such a result is easely proved in the autonomous case as the 
identity $U_{0}(t+h, s)=U_{0}(t, s-h)$ permits to use the assumption on $g$. This fails to be true in the time-depending case. For this reason, we prove the existence in $H_{m g}^{1}$ in a direct way.

2.3.1. Existence of solution for approximated problem. In the case where the magnetic potential depends on time, we can not use the method of [5] to prove existence of solution on (2.14) in $H_{m g}^{1}$. However we can prove the following.

Proposition 2.10. Let $\varphi \in H_{m g}^{1}$, then there exists $\widetilde{T}>0$ such that there exists $u_{m} \in C\left(\left[0, \widetilde{T}\left[, H_{m g}^{1}\right) \cap C^{1}\left(\left[0, \widetilde{T}\left[, H_{m g}^{-1}\right)\right.\right.\right.\right.$ solution of 2.14]). Moreover we have for all $t \in[0, \widetilde{T}]$,

$$
E_{m}\left(t, u_{m}\right)=E_{m}(t, \varphi)-\operatorname{Re} \int_{0}^{t}\left\langle\partial_{s} A(s) u(s),\left(i \nabla_{x}-A(s)\right) u(s)\right\rangle d s
$$

and

$$
\left\|u_{m}(t)\right\|_{L^{2}}=\|\varphi\|_{L^{2}} .
$$

Proof. The method consists in approximating the magnetic potential $A(t, x)$ by potentials which are piecewise constant with respect to time. More precisely, remark that thanks to Assumption 1 and Proposition 2.6 there exists $T_{2}=T_{2}(M)>0$ such that for all $t_{0} \in\left[0, T_{2}\right]$ the Cauchy problem

$$
i \partial_{t} u=\mathrm{H}_{\mathrm{A}\left(\mathrm{t}_{0}\right)} u(t)+f_{m}(u(t)), u_{\mid t=t_{0}}=\varphi
$$

can be solved in $C\left(\left[t_{0}, t_{0}+T_{2}\right], H_{A\left(t_{0}\right)}^{1}\right)$ for all initial data such that $\|\varphi\|_{H_{A\left(t_{0}\right)}^{1}} \leq M$.

Let $T \in] 0, T_{2}\left[\right.$ and for $n \in \mathbb{N}^{*}, k \in\{0, \ldots, n-1\}$ define $t_{n}^{k}=\frac{k T}{n}$. We set $A_{n}(t, x)=A\left(t_{n}^{k}, x\right), \forall t \in\left[t_{n}^{k}, t_{n}^{k+1}\left[\right.\right.$ and $A_{n}(T, x)=A(T, x)$. Next, we define the Hamiltonian $H_{n}=\left(i \nabla_{x}-A_{n}\right)^{2}$ and we look for solutions $u_{n, m}$ of

$$
i \partial_{t} u=H_{n} u+f_{m}(u), u_{\mid t=0}=\varphi .
$$

From uniqueness in the autonomous case, such a function is given by

$$
u_{n, m}(t, x)=\sum_{k=0}^{n-1} 1_{\left[t_{n}^{k}, t_{n}^{k+1}[\right.}(t) v_{k, n, m}(t, x)
$$

where $v_{k, n, m}(t, x)$ is defined as follows. We choose $v_{0, n, m}$ to be solution of

$$
\left\{\begin{array}{l}
i \partial_{t} v_{0, n, m}=\left(i \nabla_{x}-A\left(t_{n}^{0}, x\right)\right)^{2} v_{0, n, m}+f_{m}\left(v_{0, n, m}\right) \\
v_{0, n, m}\left(t_{n}^{0}, x\right)=\varphi(x)
\end{array}\right.
$$

and for $k \geq 1, v_{k, n, m}(t, x)$ is the solution of

$$
\left\{\begin{array}{c}
i \partial_{t} v_{k, n, m}=\left(i \nabla_{x}-A\left(t_{n}^{k}, x\right)\right)^{2} v_{k, n, m}+f_{m}\left(v_{k, n, m}\right) \\
v_{k, n, m}\left(t_{n}^{k}, x\right)=v_{k-1, n, m}\left(t_{n}^{k}, x\right) .
\end{array}\right.
$$

Thanks to Proposition 2.6. the function $v_{k, n, m}$ are well defined and belong to $C^{0}\left(\left[t_{n}^{k}, t_{n}^{k}+T_{2}\right], H_{m g}^{1}\right)$ and satisfy the following conservation equations

$$
E_{n, m}\left(t, v_{k, n, m}(t)\right)=E_{n, m}\left(t_{n}^{k}, v_{k, n, m}\left(t_{n}^{k}\right)\right)
$$

for all $k=0, \ldots, n-1, t \in\left[t_{n}^{k}, t_{n}^{k+1}\left[\right.\right.$ and where for all $w \in H_{m g}^{1}\left(\mathbb{R}^{n}\right)$,

$$
E_{n, m}(t, w)=\frac{1}{2} \int_{\mathbb{R}^{n}}\left|\left(i \nabla_{x}-A_{n}(t, x)\right) w(x)\right|^{2} d x-G_{m}(w) .
$$


L. MICHEL

Let us write $A\left(t_{n}^{k}, x\right)=A\left(t_{n}^{k-1}, x\right)+W_{n, k}(x)$ with $W_{n, k}(x)=\frac{1}{2} \int_{t_{n}^{k-1}}^{t_{n}^{k}} \partial_{t} A(t, x) d t$ and use $v_{k, n, m}\left(t_{n}^{k}, x\right)=v_{k-1, n, m}\left(t_{n}^{k}, x\right)$, then

$$
\begin{aligned}
E_{n, m}\left(t_{n}^{k}, u_{n, m}\left(t_{n}^{k}\right)\right) & =E_{n, m}\left(t_{n}^{k-1}, u_{n, m}\left(t_{n}^{k-1}\right)\right) \\
& -\int_{t_{n}^{k-1}}^{t_{n}^{k}} \operatorname{Re}\left\langle\left(i \nabla_{x}-A\left(t_{n}^{k-1}\right)\right) u_{n, m}\left(t_{n}^{k-1}\right), \partial_{t} A(t, x) u_{n, m}\left(t_{n}^{k-1}\right)\right\rangle d t \\
& +\left\|W_{n, k} u_{n, m}\left(t_{n}^{k-1}\right)\right\|_{L^{2}}^{2} .
\end{aligned}
$$

Thanks to Assumption 1 and conservation of mass, we have $\left\|W_{n, k} u_{n, m}\left(t_{n}^{k}\right)\right\|_{L^{2}}^{2}=$ $O\left(\frac{\|\varphi\|_{L^{2}}^{2}}{n^{2}}\right)$ uniformly with respect to $k, n, m$.

Hence, taking the sum of equations (2.22) for $k=1, \ldots, k_{0}$ with $k_{0}=\left[\frac{n t}{T}\right]$, and using the fact that the energy is constant on $\left[t_{n}^{k_{0}}, t_{n}^{k_{0}+1}\right.$ [ we get for $t \in\left[t_{n}^{k_{0}}, t_{n}^{k_{0}+1}\right.$ [

$$
\begin{aligned}
E_{n, m}\left(t, u_{n, m}(t)\right) & =E_{n, m}(0, \varphi) \\
& -\sum_{k=1}^{k_{0}} \int_{t_{n}^{k-1}}^{t_{n}^{k}} \operatorname{Re}\left\langle\left(i \nabla_{x}-A\left(t_{n}^{k-1}\right)\right) u_{n, m}\left(t_{n}^{k-1}\right), \partial_{t} A(t, x) u_{n, m}\left(t_{n}^{k-1}\right)\right\rangle d t \\
& +O\left(\frac{t}{n}\|\varphi\|_{L^{2}}^{2}\right) .
\end{aligned}
$$

With this equation we can show that the sequence $\left(u_{n, m}\right)_{(n, m) \in \mathbb{N} \times \mathbb{N}}$ is bounded in $H_{m g}^{1}$. The proof is a discretization of the proof of Lemma 3.6 in [5]. Let $M=$ $2\|\varphi\|_{H_{m g}^{1}}$ and let $T_{n, m}>0$ the maximal time such that $1+2 m_{A} T_{n, m}+m_{A}^{2} T_{n, m}^{2} \leq \frac{5}{4}$ and for $t \in\left[0, T_{n, m}[\right.$,

$$
\left\|u_{n, m}\right\|_{H_{m g}^{1}} \leq M
$$

Thanks to Propositions 1.12 .3 and Lemma 2.2 there exists $K(M)>0$ independent of $n, m \in \mathbb{N}$, such that

$$
\left\|\partial_{t} u_{n, m}\right\|_{H_{m g}^{-1}} \leq K(M), \forall n, m \in \mathbb{N}, \forall t \in\left[0, T_{n, m}[\right.
$$

and consequently,

$$
\left\|u_{n, m}-\varphi\right\|_{L^{2}} \leq 2 M K(M) t, \forall t \in\left[0, T_{n, m}[.\right.
$$

On the other hand, it follows from (2.23) that

$$
\begin{aligned}
& \frac{1}{2}\left\|\left(i \nabla_{x}-A_{n}(t)\right) u_{n, m}(t)\right\|_{L^{2}}^{2} \leq \frac{1}{2}\left\|\left(i \nabla_{x}-A(0)\right) \varphi\right\|_{L^{2}}^{2}+G_{m}\left(u_{n, m}\right)-G_{m}(\varphi) \\
& \quad-\sum_{k=1}^{n} \int_{t_{n}^{k-1}}^{t_{n}^{k}} \operatorname{Re}\left\langle\left(i \nabla_{x}-A\left(t_{n}^{k}\right)\right) u_{n, m}\left(t_{n}^{k}\right), \partial_{s} A(s, x) u_{n, m}\left(t_{n}^{k-1}\right)\right\rangle d s \\
& +O\left(\frac{t}{n}\|\varphi\|_{L^{2}}^{2}\right) .
\end{aligned}
$$

As $\partial_{t} A$ is bounded, the fourth term of the right hand side of (2.25) is bounded by $C t M^{2}$. Moreover it follows from Lemma 2.4 and estimate (2.24) that

$$
\left|G_{m}\left(u_{n, m}\right)-G_{m}(\varphi)\right| \leq C(M)\left(t+t^{\nu}\right) .
$$

Combining these equations with Proposition 1.1 we get

$$
\left\|u_{n, m}\right\|_{H_{m g}^{1}}^{2} \leq \frac{25}{16} \frac{M^{2}}{2}+C(M)\left(T_{n, m}+T_{n, m}^{\nu}\right) .
$$


Taking $0<T_{n, m}<T$ with $T$ sufficiently small independently on $n, m$, this proves that

$$
\left\|u_{n, m}(t)\right\|_{L^{\infty}\left([0, T], H_{m g}^{1}\right)} \leq M, \forall n, m \in \mathbb{N}
$$

Let now $p, q \in \mathbb{N}$, then

$i \partial_{t}\left(u_{p, m}-u_{q, m}\right)(t)=H_{p}\left(u_{p, m}-u_{q, m}\right)(t)+R_{p, q, m}(t)+g_{m}\left(u_{p, m}(t)\right)-g_{m}\left(u_{q, m}(t)\right)$

and $\left(u_{p, m}-u_{q, m}\right)_{\mid t=0}=0$, where

$$
\begin{aligned}
R_{p, q, m}(t)=\left(\left(A_{q}-A_{p}\right)(i \nabla-A(0))\right. & +(i \nabla-A(0))\left(A_{q}-A_{p}\right)(t) \\
& \left.+\left(A_{p}^{2}-A_{q}^{2}\right)(t)+2 A(0)\left(A_{q}-A_{p}\right)(t)\right) u_{q, m}(t) .
\end{aligned}
$$

Thanks to Theorem 2, we have for $\widetilde{T} \in] 0, T\left[, r \in\left[, \frac{2 n}{n-2}\left[\right.\right.\right.$ and $\frac{2}{q}=n\left(\frac{1}{2}-\frac{1}{r}\right)$,

$$
\begin{aligned}
& \left\|u_{p, m}-u_{q, m}\right\|_{L^{q}\left([0, \widetilde{T}], L^{r}\left(\mathbb{R}^{n}\right)\right)} \leq\left\|R_{p, q, m}\right\|_{L^{\infty}\left([0, \widetilde{T}], L^{2}\left(\mathbb{R}^{n}\right)\right)}
\end{aligned}
$$

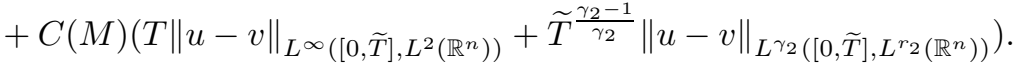

On the other hand, $\epsilon>0$ being fixed, for $p, q$ large enough we have

$$
\sup _{(t, x) \in \mathbb{R} \times \mathbb{R}^{n}}\left|A_{p}-A_{q}\right| \leq \epsilon .
$$

Hence,

$$
\left\|R_{p, q, m}\right\|_{L^{\infty}\left([0, \widetilde{T}], L^{2}\left(\mathbb{R}^{n}\right)\right)}\|\leq 2 \epsilon\| u_{q, m}\left\|_{H_{m g}^{1}}+C \epsilon\right\| u_{q, m} \|_{L^{2}} \leq C M \epsilon,
$$

and for $p, q$ large enough we get

$$
\begin{aligned}
& \left\|u_{p, m}-u_{q, m}\right\|_{L^{q}\left([0, \widetilde{T}], L^{r}\left(\mathbb{R}^{n}\right)\right)} \leq \epsilon+C(M) \widetilde{T}\|u-v\|_{L^{\infty}\left([0, \widetilde{T}], L^{2}\left(\mathbb{R}^{n}\right)\right)}
\end{aligned}
$$

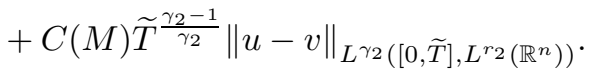

This estimate is available, both for $(q, r)=(\infty, 2)$ and $(q, r)=\left(\gamma_{2}, \rho_{2}\right)$. Summing the two inequalities obtained and making $\widetilde{T}>0$ small enough, we get

$$
\left\|u_{p, m}-u_{q, m}\right\|_{L^{q}\left([0, \widetilde{T}], L^{r}\left(\mathbb{R}^{n}\right)\right)} \leq 2 \epsilon .
$$

Therefore, the sequence $\left(u_{n, m}\right)_{n \in \mathbb{N}}$ converges, as $n$ goes to infinity, to a limit $u_{m} \in$ $L^{2}$ which is solution of (2.14). Moreover, as $\left(u_{n, m}\right)_{n \in \mathbb{N}}$ is bounded in $H_{m g}^{1}$ we can suppose that it converges weakly to $u_{m}$ in $H_{m g}^{1}$.

Now let's go back to equation (2.23). Using the fact that $u_{n, m}$ converges in $L^{2}$ and converges weakly in $H_{m g}^{1}$ it is no hard to see that $E_{n, m}\left(t, u_{n, m}\right)-E_{n, m}(0, \varphi)$ converges as $n \rightarrow \infty$, to $\operatorname{Re} \int_{0}^{t}\left\langle\partial_{s} A(s) u_{m}(s),\left(i \nabla_{x}-A(s)\right) u_{m}(s)\right\rangle d s$. From Proposition 2.3 and weak lower semicontinuity of the magnetic Sobolev norm $\|(i \nabla-A(t)) \cdot\|_{L^{2}}$ it follows that

$$
E_{m}\left(t, u_{m}\right) \leq E_{m}(0, \varphi)-\operatorname{Re} \int_{0}^{t}\left\langle\partial_{s} A(s) u(s),\left(i \nabla_{x}-A(s)\right) u(s)\right\rangle d s .
$$

Finally, $t>0$ being fixed, consider $v_{n, m}(s)=u_{n, m}(t-s)$, which is solution of

$$
i \partial_{s} v_{n, m}=-\mathrm{H}_{\mathrm{A}(\mathrm{t}-\mathrm{s})} v_{n, m}-g_{m}\left(v_{n, m}\right)
$$

with initial data $v_{n, m}(s=0)=u_{n, m}(t)$. Then we can do the same computations as above to get the converse inequality and hence (2.16) is proved. 
2.3.2. Convergence to the initial problem. In this section, we show that the sequence $u_{m}$ converges to a solution of (1.1) when $m$ goes to infinity..

Lemma 2.11. There exists $\widetilde{T}_{2}>0$ depending only on $\|\varphi\|_{H_{m g}^{1}}$ such that $\left(u_{m}\right)_{m \in \mathbb{N}}$ is a Cauchy sequence in $C\left(\left[0, \widetilde{T}_{2}\right], L^{2}\right)$.

Proof. The proof is the same as in [5], making use of Theorem 2, Lemma 2.4] Proposition 2.3 and Proposition 2.10.

Now, we can complete the proof of Theorem 1] This is the same as in [5] and we recall it for reader convenience. We denote $u$ the limit of $u_{m}$ in $C\left(\left[0, \widetilde{T}_{2}\right], L^{2}\right)$. From estimate (2.26), it follows that $u \in L^{\infty}\left(\left[0, \widetilde{T}_{2}\right], H_{m g}^{1}\right)$ and by Lemma 2.2. $u_{m}$ converges to $u$ in $C\left(\left[0, \widetilde{T}_{2}\right], L^{r}\right)$ for all $r \geq 2 n /(n-2)$. Hence, it follows from Proposition 2.3 that $f_{m}\left(u_{m}\right)$ converges to $f(u)$ in $C\left(\left[0, \widetilde{T}_{2}\right], H_{m g}^{-1}\right)$ and $u$ solves (1.1) in $L^{\infty}\left(\left[0, \widetilde{T}_{2}\right], H_{m g}^{-1}\right)$. Moreover, combining Lemma 2.4 and Proposition 2.10 we prove that

$$
E(t, u)=E(0, \varphi)-\operatorname{Re} \int_{0}^{t}\left\langle\partial_{s} A(s) u(s),\left(i \nabla_{x}-A(s)\right) u(s)\right\rangle d s .
$$

This shows that $u \in C\left(\left[0, \widetilde{T}_{2}\left[, H_{m g}^{1}\right)\right.\right.$ and hence $u \in C^{1}\left(\left[0, \widetilde{T}_{2}\left[, H_{m g}^{-1}\right)\right.\right.$.

\section{WKB APPROXIMATION}

In this section we justify WKB approximation for solution of (1.1) when the strength of the magnetic field $b$ goes to infinity and obtain instability results. We stress our attention on the case where the magnetic field and the non-linearity have the same strength; that is we consider the case $\gamma=2$ and search approximate solution for

$$
\left\{\begin{array}{l}
\left.i \partial_{s} u=\mathrm{H}_{\mathrm{A}(\mathrm{s})} u+b^{2} u g\left(|u|^{2}\right)\right) \\
u_{\mid s=0}=a_{0}(x) e^{i b S(x)}
\end{array}\right.
$$

where $g$ does not depend on $x$. Remark that with the previous notations, $f=$ $u g\left(|u|^{2}\right)$. In thi section we still ask $f$ to satisfies Assumption 2 and we require additionnaly

Assumption 3. $g \in C^{\infty}\left(\mathbb{R}_{+}, \mathbb{R}\right)$ with $g^{\prime}>0$.

Remark that if we suppose that $a_{0} \in H^{1}$ and $\nabla S+A(0) \in L^{2}$ then the initial data satisfies $\left\|a_{0}(x) e^{i b S(x)}\right\|_{H_{m g}^{1}}=O(b)$. Therefore, under Assumptions 1, 2 and 3 it follows from Theorem 1 that there exists a unique solution of (3.1) in $\left.C\left(-T_{b}, T^{b}\right], H_{m g}^{1}\right)$ with $T_{b}, T^{b} \geq C b^{-\delta}, \delta=\max \left(2, \frac{2}{\alpha}\right)$. In fact this solution takes a particular form.

Theorem 3. Let $\sigma>\frac{n}{2}+2$ and suppose that Assumptions 1 , 2 and 9 are satisfied. Assume additionally that $\partial_{t} A$ belongs to $H^{\sigma-1}\left(\mathbb{R}^{n}\right)$ for all $t \in \mathbb{R}$ and take $a_{0}$ in $H^{\sigma}\left(\mathbb{R}^{n}\right)$ and $S$ such that $\nabla S+A(t=0)$ belongs to $H^{\sigma-1}\left(\mathbb{R}^{n}\right)$. Then, there exists $T>0$ and $\alpha_{b}, \phi_{b}$ in $C\left(\left[0, T\left[, H^{\sigma}\left(\mathbb{R}^{n}\right)\right) \cap C^{1}\left(\left[0, T\left[, H^{\sigma-1}\left(\mathbb{R}^{n}\right)\right)\right.\right.\right.\right.$ such that $u(t, x)=$ $\alpha_{b}(b t, x) e^{i b\left(S(x)+\phi_{b}(b t, x)\right)}$ is solution of (3.1) on $\left[0, b^{-1} T\right]$.

Proof. We start the proof by a time rescalling leading to a semiclassical feature. We denote $h=b^{-1}>0$ and set $u(s)=v(b s)$. Then equation (3.1) is equivalent to

$$
\left\{\begin{aligned}
i h \partial_{t} v & =\left(i h \nabla_{x}-A(h t)\right)^{2} v+v g\left(|v(t)|^{2}\right) \\
v_{\mid t=0} & =a_{0}(x) e^{i h^{-1} S(x)}
\end{aligned}\right.
$$


We follow the general method initiated by Grenier 11 for the semiclassical Schrödinger equation and look for a phase and an amplitude depending on the parameter $h$. Putting the ansatz $v(t, x)=\alpha_{h}(t, x) e^{i h^{-1} \phi_{h}(t, x)}$ in the equations (3.2) we get

$$
\left\{\begin{array}{l}
\partial_{t} \phi_{h}+\left|\nabla_{\mathrm{A}} \phi_{h}\right|^{2}+g\left(\left|\alpha_{h}\right|\right)^{2}=0 \\
\partial_{t} \alpha_{h}+\nabla_{\mathrm{A}} \phi_{h} \cdot \nabla \alpha_{h}+\operatorname{div}\left(\nabla_{\mathrm{A}} \phi_{h}\right) \alpha_{h}=i h \Delta \alpha_{h}
\end{array}\right.
$$

where $\nabla_{\mathrm{A}} \phi=\left(\nabla_{x} \phi+A(h t)\right)$. Next we set $\varphi_{h}(t, x)=\nabla_{\mathrm{A}} \phi_{h}(t, x) \in \mathbb{R}^{n}$ and differentiate the above eikonal equation with respect to $x$. We obtain

$$
\left\{\begin{array}{c}
\partial_{t} \varphi_{h}+2 \varphi_{h} \cdot \nabla \varphi_{h}+2 g^{\prime}\left(\left|\alpha_{h}\right|^{2}\right) \operatorname{Re}\left(\overline{\alpha_{h}} \nabla \alpha_{h}\right)=h \partial_{t} A(h t, x) \\
\partial_{t} \alpha_{h}+\varphi_{h} \cdot \nabla \alpha_{h}+\operatorname{div}\left(\varphi_{h}\right) \alpha_{h}=i h \Delta \alpha_{h}
\end{array}\right.
$$

Separating real and imaginary parts of $\alpha_{h}=\alpha_{1, h}+i \alpha_{2, h}$, (3.4) becomes

$$
\partial_{t} w_{h}+\sum_{j=1}^{n} A_{j}\left(w_{h}\right) \partial_{x_{j}} w_{h}=h L w_{h}+\nu_{h}
$$

with

$$
\begin{gathered}
w_{h}=\left(\begin{array}{c}
\alpha_{1, h} \\
\alpha_{2, h} \\
\varphi_{1, h} \\
\vdots \\
\varphi_{n, h}
\end{array}\right), \nu_{h}=\left(\begin{array}{c}
0 \\
0 \\
h \partial_{t} A_{1}(h t, x) \\
\vdots \\
h \partial_{t} A_{n}(h t, x)
\end{array}\right) \\
L=\left(\begin{array}{ccc}
0 & -\Delta & 0 \\
\Delta & 0 & 0 \\
0 & 0 & 0_{n \times n}
\end{array}\right)
\end{gathered}
$$

and

$$
A_{j}(w)=\left(\begin{array}{ccccc}
\varphi_{j, h} & 0 & \alpha_{1} & \ldots & \alpha_{1} \\
0 & \varphi_{j, h} & \alpha_{2} & \ldots & \alpha_{2} \\
2 g^{\prime} \alpha_{1} & 2 g^{\prime} \alpha_{2} & v_{j} & 0 & 0 \\
\vdots & \vdots & 0 & \ddots & 0 \\
2 g^{\prime} \alpha_{1} & 2 g^{\prime} \alpha_{2} & 0 & 0 & \varphi_{j, h}
\end{array}\right)
$$

This system has the same form as in [1], 3] with the exception of the source term $\nu_{h}$ in right hand side of (3.5) and the initial data. Thanks to the assumptions, $\nu_{h}$ belongs to $H^{\sigma-1}\left(R^{n}\right)$, whereas the initial condition in (3.2) yields

$$
w_{h}(t=0)=\left(\begin{array}{c}
\operatorname{Re} a_{o} \\
\operatorname{Im} a_{0} \\
\partial_{x_{1}} S+A_{1}(0) \\
\vdots \\
\partial_{x_{n}} S+A_{n}(0)
\end{array}\right)
$$

which belongs to $H^{\sigma-1}\left(\mathbb{R}^{n}\right)$.

On the other hand, thanks to the assumption on $g^{\prime}$, the system (3.5) can be symmetrized by

$$
S=\left(\begin{array}{cc}
I_{2} & 0 \\
0 & \frac{1}{g^{\prime}} I_{n}
\end{array}\right)
$$


which is symmetric and positive. It follows from general theory of hyperbolic systems that the problem (3.5) together with initial condition (3.9) has a unique solution $w_{h} \in L^{\infty}\left(\left[0, T_{h}\right], H^{\sigma-1}\right)$ for some $T_{h}>0$.

Hence, we have to bound $T_{h}$ from below by a constant independent of $h$. This is done by computing classical energies estimates as in [11, [3], and using the fact that $\partial_{t} A$ as well as $\nabla_{x} S+A(0)$ belong to $H^{\sigma-1}$.

Finally we define $\alpha_{h}$ and $\phi_{h}$ by $\alpha_{h}=w_{1, h}+i w_{2, h}$ and

$$
\phi_{h}=S(x)-\int_{0}^{t}\left|\varphi_{h}\right|^{2}+f\left(\left|\alpha_{h}\right|^{2}\right) d s .
$$

By construction, $\phi_{h}$ belongs to $L^{2}$. Moreover, a simple calculus shows that $\nabla_{x} \phi_{h}=$ $\varphi_{h}-A(h t)$ belongs to $H^{\sigma-1}$ so that $\phi_{h}$ is in fact in $H^{\sigma}$. Going back to the equation on $\alpha_{h}$ and making energies estimates we show that $\alpha_{h} \in H^{\sigma}$. Finally, it a direct calculus shows that $\left(\alpha_{h}, \phi_{h}\right)$ defined above solves (3.3)

Remark 3.1. The above solution belongs to the magnetic sobolev space $H_{m g}^{1}$. Indeed,

$$
\left(i \nabla_{x}-b A\right)\left(\alpha_{b} e^{i b \phi_{b}}\right)=\left(i \nabla \alpha_{b}-b\left(\nabla \phi_{b}+A\right) \alpha_{b}\right) e^{i b \phi_{b}}
$$

belongs to $L^{2}$. Therefore the solution built in Theorem 1 coincide with the one of Theorem 1 .

With Theorem 3 in hand it is easy to prove instability results.

Proposition 3.2. Let $\sigma>\frac{n}{2}+2$ and let $A$ satisfy the assumptions of Theorem 3. Suppose that $S$ is such that $\nabla S+A(t=0)$ belongs to $H^{\sigma-1}\left(\mathbb{R}^{n}\right)$. Then, there exists $a_{0}$ and $\widetilde{a}_{0, b}$ in $H^{\sigma}\left(\mathbb{R}^{n}\right)$ and $0<t_{b}<C b^{-1}$ such that

$$
\left\|a_{0}-\widetilde{a}_{0, b}\right\|_{L^{2}} \rightarrow 0 \text { as } b \rightarrow \infty
$$

and the solutions $u_{b}$ (resp. $\widetilde{u}_{b}$ ) associated to (3.1) with initial data $a_{0} e^{i b S(x)}$ (resp. $\left.\widetilde{a}_{0} e^{i b S(x)}\right)$ satisfy

$$
\left\|u_{b}-\widetilde{u}_{b}\right\|_{L^{\infty}\left(\left[0, t_{b}\right], L^{2}\right)} \geq 1 .
$$

Proof. It is a straightforward consequence of Theorem $[3$ and the methods of $[3]$.

\section{REFERENCES}

1. J. Avron, I. Herbst, and B. Simon, Schrödinger operators with magnetic fields. I. General interactions, Duke Math. J. 45 (1978), no. 4, 847-883.

2. N. Burq, P. Gérard, and N. Tzvetkov, On nonlinear Schrödinger equations in exterior domains, Ann. Inst. H. Poincaré Anal. Non Linéaire 21 (2004), no. 3, 295-318.

3. R. Carles, Geometric optics and instability for semi-classical schrödinger equations, Arch. Ration. Mech. Anal. (to appear).

4. T. Cazenave and M. J. Esteban, On the stability of stationary states for nonlinear Schrödinger equations with an external magnetic field, Mat. Apl. Comput. 7 (1988), no. 3, 155-168.

5. T. Cazenave and F. B. Weissler, The Cauchy problem for the nonlinear Schrödinger equation in $H^{1}$, Manuscripta Math. 61 (1988), no. 4, 477-494.

6. M. Christ and A. Kiselev, Maximal functions associated to filtrations, J. Funct. Anal. 179 (2001), no. 2, 409-425.

7. A. De Bouard, Nonlinear Schroedinger equations with magnetic fields, Differential Integral Equations 4 (1991), no. 1, 73-88.

8. V. Georgiev and M. Tarulli, Scale invariant energy smoothing estimates for the Schrödinger equation with small magnetic potential, Asymptot. Anal. 47 (2006), no. 1-2, 107-138. 
9. J. Ginibre and G. Velo, The classical field limit of scattering theory for nonrelativistic manyboson systems. II, Comm. Math. Phys. 68 (1979), no. 1, 45-68.

10. - The global Cauchy problem for the nonlinear Schrödinger equation revisited, Ann. Inst. H. Poincaré Anal. Non Linéaire 2 (1985), no. 4, 309-327.

11. E. Grenier, Semiclassical limit of the nonlinear Schrödinger equation in small time, Proc. Amer. Math. Soc. 126 (1998), no. 2, 523-530.

12. Ginibre J. and Velo G., Long range scattering for some schrödinger related nonlinear systems, preprint.

13. M. Nakamura and T. Wada, Local well-posedness for the Maxwell-Schrödinger equation, Math. Ann. 332 (2005), no. 3, 565-604.

14. Y. Nakamura and A. Shimomura, Local well-posedness and smoothing effects of strong solutions for nonlinear Schrödinger equations with potentials and magnetic fields, Hokkaido Math. J. 34 (2005), no. 1, 37-63.

15. A. Pazy, Semigroups of linear operators and applications to partial differential equations, Applied Mathematical Sciences, vol. 44, Springer-Verlag, New York, 1983.

16. A. Stefanov, Strichartz estimates for the magnetic schrdinger equation, http://arxiv.org/abs/math.AP/0509314 (2005).

17. Y. Tsutsumi, Global existence and asymptotic behavior of solutions for the MaxwellSchrödinger equations in three space dimensions, Comm. Math. Phys. 151 (1993), no. 3, 543-576.

18. K. Yajima, Schrödinger evolution equations with magnetic fields, J. Analyse Math. 56 (1991), $29-76$.

(L. Michel) Laboratoire J. A. Dieudonné, Université de Nice Sophia-Antipolis, ParC Valrose, 06108 Nice Cedex 02, France

E-mail address, L. Michel: lmichel@math.unice.fr 